\title{
How Does Adhesion Induce the Formation of Telephone Cord Buckles?
}

\author{
Jean-Yvon Faou, ${ }^{1, *}$ Guillaume Parry, ${ }^{2, \dagger}$ Sergey Grachev, ${ }^{1}$ and Etienne Barthel ${ }^{1}$ \\ ${ }^{1}$ Surface du Verre et Interfaces, UMR 125 CNRS/Saint-Gobain, 39 Quai Lucien Lefranc, F-93303 Aubervilliers, Cedex, France \\ ${ }^{2}$ SIMaP, Grenoble INP-CNR-UJF, BP. 75, 38402 Saint Martin d'Hères cedex, France
}

(Received 4 December 2011; revised manuscript received 11 January 2012; published 12 March 2012)

\begin{abstract}
Compressively stressed thin films with low adhesion frequently buckle and delaminate simultaneously into telephone cords. Although these buckles have been studied for decades, no complete understanding of their propagation has so far been presented. In this study, we have coupled a nonlinear plate deformation with a cohesive zone model to simulate the kinematics of a propagating telephone cord buckle in very close agreement with experimental observations. Proper inclusion of the dependence of an adhesion upon the mode mixity proved to be central to the success of the approach. The clarification of the mechanism promises better understanding of buckle morphologies.
\end{abstract}

DOI: 10.1103/PhysRevLett.108.116102

PACS numbers: $68.60 . \mathrm{Bs}, 46.32 .+\mathrm{x}, 62.25 . \mathrm{Mn}$

Thin films and coatings are widely used in many hightech applications such as low emissivity windows [1], thermal barrier coatings for aeronautical applications [2], and microelectronic devices [3]. In such systems, large compressive residual stresses often reside in the films, causing buckling and delamination, which is detrimental to the application. Therefore, the buckling and delamination of stressed multilayers have been widely investigated in the framework of material science [4,5] and mainly of plate mechanics [6-10]. The versatile morphologies of buckles, which may appear in a wide variety of shapes, namely, straight, circular, telephone cord (TC), and more, have been the topic of in-depth investigations based on nonlinear thin plate theory. Analytical approaches based on the Von Kármán theory of plates have provided valuable insights into straight-sided blisters, for which the coupled post-buckling elastic deformation and delamination problems are analytically tractable [11]. More recently, it was established that the TC buckles, as well as some circular shapes, could be viewed as equilibrium configurations evolving out of the formation of straight-sided blisters, a phenomenon known as "secondary buckling" [12-16]. The rich morphology and the transitions between these equilibria have also been studied in detail $[17,18]$. However, these results do not take into account the additional complexities involved in interfacial toughness and thus do not explain the formation of TC buckles in adherent films. It has been shown by experimental studies that interfaces are usually significantly more difficult to break by applying shear loading, that is, in mode II, than by normal forces, that is, in mode I $[19,20]$. This is why the mode mixity (connected to the ratio between the two modes) at the front of the blister has to be considered in detail. In a straight-sided blister, the mode II contribution increases with respect to mode I as the blister becomes wider [11]. At some point, as the loading at the sides becomes pure mode II, the lateral expansion of the straight-sided blister stops. However, at the extremities of a straight-sided blister, the loading never reaches pure mode II and the blister can propagate indefinitely along its longitudinal axis. The energy released due to the relaxation of the stress in the film is then used to fracture the interface. For more complex morphologies, it has been shown that the amount of energy released depends on the blister morphology [21], while the mode mixity at the crack front determines the energy needed to fracture the interface. However, to date, calculations have only investigated the influence of mode mixity on static, final configurations [22], although it is precisely the coupling between interface response and transient buckle shapes that accounts for the propagation and, in the end, explains the morphology of the buckles. This more complex task has not been achieved previously and this is why the propagation of a buckle and the origin of even the ubiquitous TC morphology is still elusive.

It has been suggested earlier that buckle propagation and especially the TC morphology is "closely tied to the configurational instability of the crack front" [23]. In this Letter, we demonstrate this mechanism: a specific TC morphology is experimentally identified, where a sag in the buckle appears immediately before the TC rotation changes direction. Sags of the same nature have been observed in propagating buckles previously [7,23]. Here, from numerical simulations, we provide evidence that this sagged area induces a pinning of the buckle front in pure shear. It is from this pinned zone that the counter-rotating branch emerges. We believe that the pinning of the front edge by shear following the buckle front collapse is actually the generic mechanism from which TC propagation results.

The samples were molybdenum coatings deposited by dc magnetron sputtering on $300 \mu \mathrm{m}$ thick silicon wafers with a native oxide layer. The base pressure prior to deposition was $4 \times 10^{-10}$ bar. The sputtering gas was argon with a flow of $40 \mathrm{sccm}$ which resulted in a pressure of $2 \times 10^{-6}$ bar. Interface toughness can be adjusted 
through interfacial layers [24]. Here, a $10 \mathrm{~nm}$ thick silver layer was deposited onto the substrate in order to ensure a relatively low adhesion, $G_{1}<1 \mathrm{~J} / \mathrm{m}^{2}$. Immediately following, a $120 \mathrm{~nm}$ thick layer of partially oxidized molybdenum, obtained by adding $2 \mathrm{sccm}$ of $\mathrm{O}_{2}$, was deposited. Controlling the oxygen content in the film leads to a fine tuning of the residual stress. The latter, measured in-situ with a multibeam optical stress sensor [25], amounted to $2.7 \pm 0.05 \mathrm{GPa}$.

These stacks can delaminate when the sample is brought to atmospheric pressure. By varying the deposition parameters, various buckle morphologies can be obtained. For the present samples, the delamination occurred at the interface between silver and the native silicon dioxide of the substrate [26] and the kinematics of buckle formation was easily recorded by optical microscopy, a $29 \mu \mathrm{m}$ wide TC progressing over one wavelength in about $28 \mathrm{~s}$. The buckle propagation was consistent with usual observations [27], with one notable addition, however: It was observed that the tip of the semicircular buckle sags when the TC completes one half-rotation and the front caves in (Fig. 1, see the Supplemental Material for a movie of the buckle propagation [28]). This sagged area is reminiscent of the configurational instabilities predicted earlier [23], and we then proceeded to identify the mechanism by finite element modeling (FEM).

To analyze this behavior, it is essential to use a nonlinear plate model to capture the buckling equilibria. The surface of the plate is defined as the $O, x, y)$ plane and the out-ofplane displacement is $w(x, y)$ (Fig. 2). In order to take into account the presence of the substrate, the unilateral contact condition, $w(x, y) \geq 0$, is introduced. The calculations are made for large displacements $w$, using the Green Lagrange strain tensor. When $w$ is large, the strain tensor reduces to

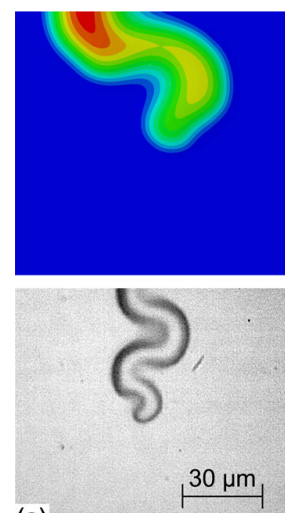

(a)

FIG. 1 (color online). Telephone cord propagation: comparison between numerical results (top) and optical measurements (bottom). Configurational instability induces a sag at the front, resulting in the pinning of the outer edge of the buckle in almost pure shear. The blister grows further around this pinning point thus changing the direction of rotation.

$$
\begin{aligned}
& e_{x x}=\frac{\partial u}{\partial x}+\frac{1}{2}\left(\frac{\partial w}{\partial x}\right)^{2}, \\
& e_{y y}=\frac{\partial v}{\partial y}+\frac{1}{2}\left(\frac{\partial w}{\partial y}\right)^{2}, \\
& e_{x y}=\frac{1}{2}\left(\frac{\partial u}{\partial y}+\frac{\partial v}{\partial x}\right)+\frac{1}{2} \frac{\partial w}{\partial x} \frac{\partial w}{\partial y} .
\end{aligned}
$$

In Eq. (1) the terms that are nonlinear in $w$ are responsible for a third order term in the thin plate equilibrium equations and it is this nonlinearity which is essential to capture the post-buckling evolution of the blisters (e.g., see [29]).

The second key point is the description of adhesion rupture for which a cohesive zone model [30-33] is used. Cohesive elements are inserted at the interface and the tractions they develop at the edges of the buckle provide boundary conditions for the buckled plate. In a reversible cohesive element, when the relative normal displacement $\delta_{n}$ between opposite crack faces increases (Fig. 2), the normal traction $T_{n}$ increases (Fig. 3). The normal traction reaches the cohesive traction $T_{n}^{0}$ (the maximum traction during interface separation) for $\delta_{n}=\delta_{n}^{0}$. Subsequently, the traction decreases and vanishes when $\delta_{n}$ reaches $\delta_{n}{ }^{f}$, the displacement at full rupture. In most cohesive zone models, simple traction-separation laws are assumed: here the traction is linear with displacement both in the increasing and decreasing regimes (Fig. 3), so that the total work of rupture is $G_{I}^{c}=1 / 2 T_{n}^{0} \delta_{n}{ }^{f}$. To implement irreversible decohesion, we allow for the cohesive traction $T_{n}^{0}$ to irreversibly decrease along the traction-separation curve as the maximum interfacial displacement $\delta_{n}$ max increases from $\delta_{n}{ }^{0}$ to $\delta_{n}{ }^{f}$ : This process reflects interface damage. After opening up to $\delta_{n}{ }^{\max }>\delta_{n}{ }^{0}$, if the cohesive element subsequently closes, it unloads from $\delta_{n}$ max to 0 with decreasing interface traction $T_{n}=K(1-d) \delta_{n}$ (Fig. 3, dashed line), where $K=T_{n}{ }^{0} / \delta_{n}{ }^{0}$ is the initial loading stiffness. The damage variable $d$ is so chosen as to increase monotonically from 0 to 1 with $\delta_{n}{ }^{\max }>\delta_{n}{ }^{0}$ and fulfill the condition $T_{n}^{0}\left(\delta_{n}^{\max }\right)=K(1-d) \delta_{n}^{\max }$. This softening of the loading-unloading segment results in irreversibility through incomplete restitution of interfacial energy upon

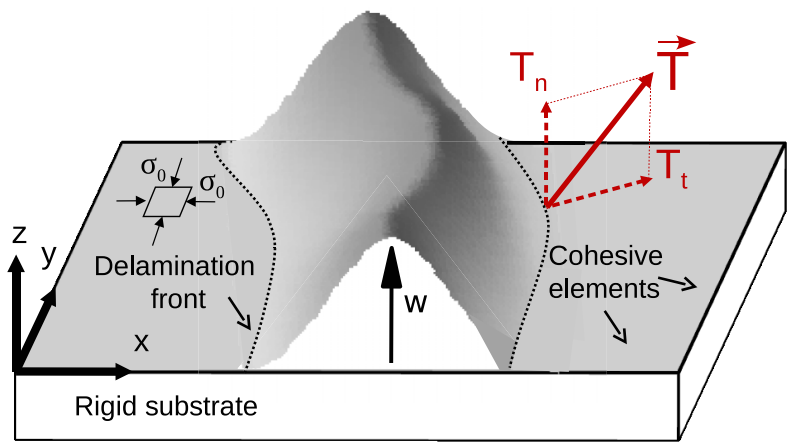

FIG. 2 (color online). Model for thin film buckling and delamination: a nonlinear plate on a rigid substrate with a mixedmode cohesive zone model for the adhesive interface. 


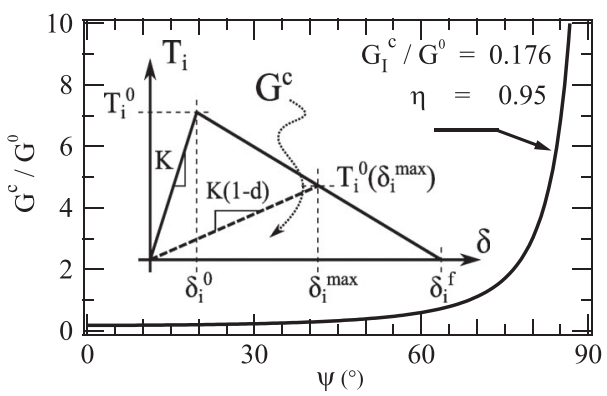

FIG. 3. Mixed-mode dependence of the work of adhesion at the film-substrate interface and bilinear traction vs separation law (Inset: $i=n$ for mode I and $t$ for mode II).

the unloading of the cohesive element. If $\delta_{n}{ }^{\text {max }}$ reaches $\delta_{n}{ }^{f}$, then $d=1, T_{n}{ }^{0}\left(\delta_{n}{ }^{f}\right)=0$ and rupture is complete. In this case, if the surfaces are brought back into contact, simple frictionless unilateral contact is enforced. A similar model applies for pure mode II, with work of adhesion $G_{\mathrm{II}}^{c}$ and cohesive traction $T_{t}^{0}$

For mixed-mode loadings, rupture may initiate for stress levels below the individual cohesive tractions, so that we use a quadratic rupture initiation criterion $\left(T_{n} / T_{n}{ }^{0}\right)^{2}+$ $\left(T_{t} / T_{t}^{0}\right)^{2}=1$. Denoting by $T_{n}^{0^{\prime}}$ and $T_{t}^{0^{\prime}}$ the stress values for which rupture initiates, we define the mode mixity angle $\psi$ by $\tan (\psi)=\left(T_{t}^{0^{\prime}} / T_{n}^{0^{\prime}}\right)$. The energy release rate is evaluated by $G^{c}(\psi)=G_{I}{ }^{c}\left[1+\tan ^{2}(\eta \psi)\right]$ [11], where $\eta$ is determined by $G^{c}(\pi / 2)=G_{\mathrm{II}}^{c}$ (Fig. 3) and is expressed as $G^{c}(\psi)=1 / 2 T_{n}{ }^{0^{\prime}} \delta_{n}{ }^{f}+1 / 2 T_{t}^{0^{\prime}} \delta_{t}{ }^{f}$ as a function of the mechanical variables. For the damage variable $d$ we define the effective cohesive stress $T_{\text {eff }}{ }^{0^{\prime}}=$ $\sqrt{\left(T_{n} 0^{\prime}\right)^{2}+\left(T_{t}^{0^{\prime}}\right)^{2}}$, the effective interfacial deformation $\delta_{\text {eff }}=\sqrt{\left(\delta_{n}\right)^{2}+\left(\delta_{t}\right)^{2}}$, and the effective deformation at full rupture $\delta_{\text {eff }}^{f}=2 G^{c}(\psi) / T_{\text {eff }} 0^{\prime}$, which is compatible with the previous expression for $G^{c}(\psi)$ if $\delta_{n}{ }^{f} / \delta_{t}{ }^{f}=$ $T_{n}{ }^{0^{\prime}} / T_{t} 0^{0^{\prime}}$. The damage variable $d$ is then defined as above, substituting the effective variable $\delta_{\text {eff }}$ for $\delta_{n}$. We have checked that this model satisfactorily reproduces the analytical predictions for the simpler geometry of the straight blister (see Supplemental Material [28]).

For telephone cords, the loading consists of an eigenstrain $\epsilon_{0}>0$ applied uniformly to the plate $\left(\epsilon_{x x}=\epsilon_{y y}=\right.$ $\left.\epsilon_{0}, \epsilon_{x y}=0\right)$. An equibiaxial compressive stress state is generated in the film: $\sigma_{x x}=\sigma_{y y}=-E /(1-\nu) \epsilon_{0}=$ $-\sigma_{0}, \sigma_{x y}=0$ (see Fig. 2). A small rectangular domain on one side remains adhesion-free: This is the initiation zone where the blister can first nucleate (Fig. 4). The FEM calculations are carried out using the software ABAQUS [34] with an implicit formulation. Quadrilateral shell elements are used. The element size is chosen so that there are about 40 elements in the buckle width.

Material models exhibiting a softening behavior, as the interface model used in this study, often result in serious

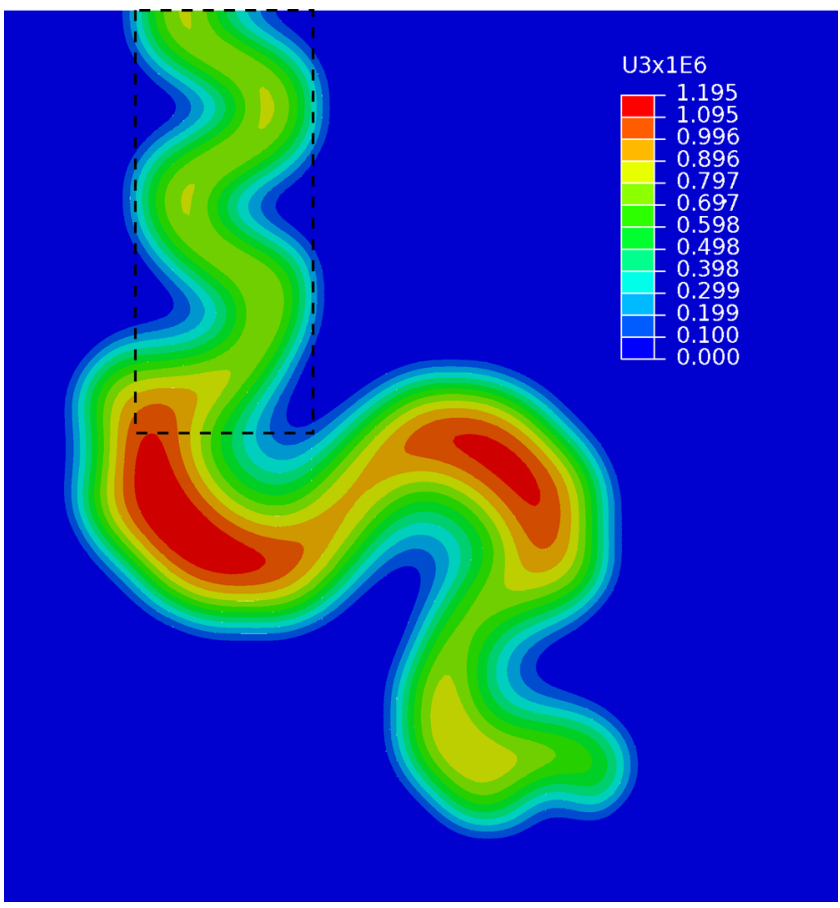

FIG. 4 (color online). Overview of the deformed shape of the delaminated film in the numerical simulation. Note the strong contrast between the buckle morphology in the adhesionless area (initiation zone, dashed line) and the morphology of the buckle propagating in the adhesive area.

convergence issues in FEM simulations. In an implicit formulation, the radius of convergence of the NewtonRaphson scheme reduces to zero at the point of instability. We avoid these issues by introducing viscous regularization of the damage variable with a characteristic time $\mu$ [34]. Viscous regularization causes the tangent stiffness matrix of the softening material to be positive for sufficiently small time increments. We checked that viscous regularization is effective if $\mu$ is taken somewhat smaller than the time step. Then viscous dissipation energy is less than $4 \%$ of the work of adhesion $G^{c}$, the tractionseparation law stays consistent at all points of the interface, and the kinematics of the propagation is independent of $\mu$ (see Supplemental Material [28]).

We chose the following values for the film: eigenstrain $\epsilon_{0}=0.18 \%$, elastic modulus $E_{f}=329 \mathrm{GPa}$, Poisson ratio $\nu_{f}=0.3$, and thickness $h=150 \mathrm{~nm}$. In-plane dimensions were normalized to $b_{0}=\pi\left\{E_{f} /\left[12\left(1-\nu_{f}^{2}\right) \sigma_{0}\right]\right\}^{1 / 2} h$. The lateral dimensions were $35 b_{0} \times 45 b_{0}$. Denoting by $G_{0}=$ $\left(1-\nu_{f}\right) h \sigma_{0}^{2} / E_{f}$, the elastic energy stored in the film, the works of adhesion were $G_{\mathrm{I}}^{c} / G_{0}=0.176$ and $G_{\mathrm{II}}^{c} / G_{0}=$ 28.5, which amounts to $\eta=0.95$ (see Fig. 3). The cohesive tractions were $T_{n}^{0}=0.02 \sigma_{0}$ and $T_{t}^{0}=0.24 \sigma_{0}$, the stiffness $K=6.6 \times 10^{2}\left(T_{n}^{0}\right)^{2} / G_{0}$. A mapping of the outof-plane displacement $w(x, y)$ during propagation is shown in Fig. 4. In the adhesion-free initiation area (dashed line), a standard TC buckle initiates readily $[13,18]$. The buckle 


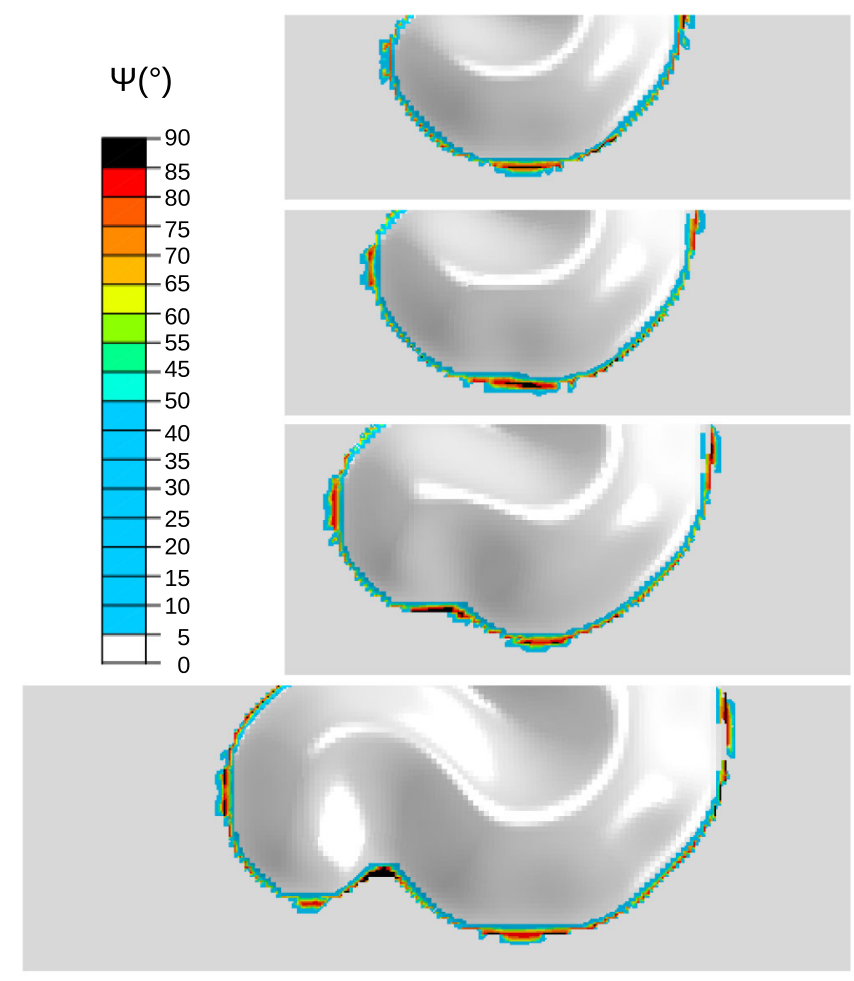

FIG. 5 (color online). Phase angle along the delamination front for four different stages of propagation. We observe that when the front grows, the configurational instability induces a large mode mixity locally and effectively pins the front. Note the slight drift of the pinning point while the buckle expands around it.

subsequently propagates to the area where the film adheres to the substrate. In Fig. 4 we show the TC after it has propagated more than one full wavelength in the adhesive area. The morphology of the TC there differs markedly from the morphology in the adhesion-free area as it is dictated not only by the film thickness and the elastic properties of the film but also by the fracture energy $G_{c}(\psi)$. With the present choice of simulation parameters, the TC propagation emulates the experiment very well [Fig. 1(a); see also the Supplemental Material for a movie of the simulated propagation [28] ]. In Fig. 1(a) the buckle grows as a counterclockwise segment of the pinned circular blister [35] with an increasing radius. Subsequently [Fig. 1(b)], the initial convex front becomes unstable and a sag appears on the outer edge of the buckle, slightly behind the front, and gives rise to a bifurcation of the propagating front into two branches. While the righthand branch gradually comes to a halt, the left-hand branch moves forward [Fig. 1(c)], turning around a specific point located near the sagged area and growing clockwise as another segment of the pinned circular blister with a growing radius.

A detailed series of snapshots of the TC buckle propagation around the turning point is shown in Fig. 5 where the mode mixity angle $\psi$ is reported around the delamination front at different stages. When the initial portion of the pinned circular blister collapses, forming the sag due to configurational instability [23], it can be observed that shear increases markedly at the interface since $\psi$ rises from its mean value of $72^{\circ}$ to almost $90^{\circ}$ (i.e., near mode II). This observation clearly demonstrates that the coupling between instability and mode mixity dependent adhesion results in the formation of a pinning point, as it is effectively impossible to break the interface in mode II. The solution of expanding around the pinning point becomes energetically more favorable for the buckle, and the delamination front then divides into two branches, expanding on each side of the pinning point. On the right-hand side of the pinning point, the previous blister keeps growing, increasing its radius into a full half-circle but stops gradually. It shifts the pinning point slightly in the process. On the left-hand side, the other front grows as a new segment of a pinned circular blister. This smaller blister grows faster than the previous one and it is this counterrotating pinned circular blister which emerges as a new TC segment. From this point, the story repeats itself.

In summary, we have demonstrated that in this specific case, telephone cord propagation originates from the pinning of the buckle front in mode II. A configurational instability appears on the outer edge of the buckle front, followed by the development of a strong mode II area, which forms the pinning point responsible for the inversion of the curvature of the buckle and finally, telephone cord propagation. A similar phenomenon is likely to apply in the general case of telephone cord propagation and we suggest that the same pinning mechanism is effective even if not directly evidenced by a sagging area. We expect that accurate simulations of TC propagation through coupled buckling delamination will make it possible to better characterize interfacial toughness and its dependence upon mode mixity in relation to specific interfaces. Finally, we can anticipate that various other buckle morphologies could be demonstrated and their formation mechanism elucidated through the present modeling strategy.

\section{*Jean-Yvon.Faou@saint-gobain.com} †uillaume.parry@simap.grenoble-inp.fr

[1] B. Karlsson, E. Valkonen, T. Karlsson, and C.-G. Ribbing, Thin Solid Films 86, 91 (1981).

[2] S. Faulhaber, C. Mercer, M.-W. Moon, J. W. Hutchinson, and A. G. Evans, J. Mech. Phys. Solids 54, 1004 (2006).

[3] O. van der Sluis, A. A. Abdallah, P. C. P. Bouten, P. H. M. Timmermans, J. M. J. den Toonder, and G. de With, Eng. Fract. Mech. 78, 877 (2011).

[4] G. Gilles and B. Rau, Thin Solid Films 120, 109 (1984).

[5] E. Spiecker, A. K. Schmid, A. M. Minor, U. Dahmen, S. Hollensteiner, and W. Jäger, Phys. Rev. Lett. 96, 086401 (2006).

[6] J. S. Wang and A. G. Evans, Acta Mater. 47, 699 (1999). 
[7] M. D. Thouless, J. Am. Ceram. Soc. 76, 2936 (1993).

[8] A. G. Evans and J. W. Hutchinson, Int. J. Solids Struct. 20, 455 (1984).

[9] J. W. Hutchinson, J. Mech. Phys. Solids 49, 1847 (2001).

[10] M. S. Hu, M.D. Thouless, and A. G. Evans, Acta Metall. 36, 1301 (1988).

[11] J.W. Hutchinson and Z. Suo, Adv. Appl. Mech. 29, 63 (1991).

[12] B. Audoly, Phys. Rev. Lett. 83, 4124 (1999).

[13] J. Colin, F. Cleymand, C. Coupeau, and J. Grilhé, Philos. Mag. A 80, 2559 (2000).

[14] M. George, C. Coupeau, J. Colin, F. Cleymand, and J. Grilhé, Philos. Mag. A 82, 633 (2002).

[15] G. Parry, C. Coupeau, J. Colin, and J. Grilhé, Acta Mater. 52, 3959 (2004).

[16] P. Waters and A. A. Volinsky, Exp. Mech. 47, 163 (2007).

[17] B. Audoly, B. Roman, and A. Pocheau, Eur. Phys. J. B 27, 7 (2002).

[18] G. Parry, A. Cimetiere, C. Coupeau, J. Colin, and J. Grilhe, Phys. Rev. E 74, 066601 (2006).

[19] K. M. Liechti and Y.S. Chai, J. Appl. Mech. 59, 295 (1992).

[20] A. Lee, C. S. Litteken, R. H. Dauskardt, and W. D. Nix, Acta Mater. 53, 609 (2005).

[21] M.-W. Moon, K.-R. Lee, K. H. Oh, and J. W. Hutchinson, Acta Mater. 52, 3151 (2004).

[22] M. J. Cordill, D.F. Bahr, N.R. Moody, and W.W. Gerberich, Mater. Sci. Eng. A 443, 150 (2007).
[23] J. W. Hutchinson, M. D. Thouless, and E. G. Liniger, Acta Metall. Mater. 40, 295 (1992).

[24] E. Barthel, O. Kerjan, P. Nael, and N. Nadaud, Thin Solid Films 473, 272 (2005).

[25] J. Floro, S. J. Hearne, J. Hunter, P. Kotula, E. Chason, S. C. Seel, and C. V. Thompson, J. Appl. Phys. 89, 4886 (2001).

[26] S. Grachev, A. Mehlich, J.-D. Kamminga, E. Barthel, and E. Sondergard, Thin Solid Films 518, 6052 (2010).

[27] A. A. Abdallah, P. C.P. Bouten, J. M. J. den Toonder, and G. de With, Thin Solid Films 516, 1063 (2008).

[28] See Supplemental Material http://link.aps.org/ supplemental/10.1103/PhysRevLett.108.116102 for movies of the observed and simulated propagations along with details of the cohesive zone model and its application.

[29] L.D. Landau and E.M. Lifshitz, Theory of Elasticity (Butterworth Heinemann, 1986).

[30] X. P. Xu and A. Needleman, Model. Simul. Mater. Sci. Eng. 1, 111 (1993).

[31] V. Tvergaard and J. W. Hutchinson, J. Mech. Phys. Solids 40, 1377 (1992).

[32] E. Barthel, J. Colloid Interface Sci. 200, 7 (1998).

[33] O. van der Sluis, A. A. Abdallah, P.C.P. Bouten, P. H. M. Timmermans, J. M. J. den Toonder, and G. de With, Eng. Fract. Mech. 78, 877 (2011).

[34] ABAQus Manuals Collection, Dassault Systèmes Simulia Corp., Providence, RI, USA (2010).

[35] M.-W. Moon, H. M. Jensen, J.W. Hutchinson, K. H. Oh, and A. G. Evans, J. Mech. Phys. Solids 50, 2355 (2002). 